\title{
The division and bending of green and red semiconductor laser light at the same time
}

\author{
Remzi Yildirim
}

Department of Computer, Gazi University, Ankara, Turkey; remzi@gazi.edu.tr, remzi1963@gmail.com

Received 19 August 2011; revised 21 September 2011; accepted 30 September 2011.

\begin{abstract}
In this experimental study, the laser light was split and bended using a specially designed glass lenses at the same time. This process has been done at the atmospheric pressure and room temperature conditions. During the experiments, the semiconductor laser as a source of green and red laser diode is used. In addition, polarization, magnetic field, electric field or any other auxiliary materials and systems which affect laser light are not used to bend the laser light. Only transparent glass lenses that are designed specially are used in the experimental study.
\end{abstract}

Keywords: Laser; Bending; Curve; Bending of Light; Bending of Laser Light

\section{INTRODUCTION}

The first studies about the light are based on spatial connection and movement reciprocally. This idea led researchers to create many theories. Aristotle and Copernican had the idea that world revolve around the sun. After that, the Kepler, Galileo, Descartes, Huygen and Newton had theories. New theories of relativity do not only include relativity principals, also they have the principle of the kinematics. These are based on the dynamic relationship between the theories have emerged. They are regularly in the Newton's mechanical laws of motion of mechanical systems has been a revolution in science is an axis placement. Later in the 19th electromagnetism century, the emergence of a new era opened in physics. In the nineteenth century to the light and the waves opened a new era on the play-warranted. In light of these studies, theories began to emerge about the speed of light is variable. Lorenz and Poincare in 1905 related to the study of relativity reached satisfactory results. This affected the outcomes in the same way and as a result of mechanical and optic revealed the real coordinate system.

*Note: The study details of the lenses are not given, because it is in the process of patent. For more picture: http://w3.gazi.edu.tr/ remzi
Then the Maxwell equations electromagnetisms described the same bi-grass. This work was also included in the speed of light. In the same period, Einstein, the Lorentz transformation based on the theory of relativity combined with the speed of light. As a result, cases have emerged electrodynamics. Into the Minkowski spacetime in 1907, and this expression has developed a generalized Pythagorean and simply. Planck theorem of relativity, Laue, Lewis, Tolman, and other scientists contributed. Einstein's theory of relativity in 1915 by re-editing has been added to the theory of gravity. There are many theory of relativity. Maxwell's equations, electric and magnetic field forces, laws and developed force showed the effect of small masses. In addition, the effect of gravitational force, masses were simply the effect of gravity. After an analysis of Newton's gravitational 20th more detailed studies of space activities began in the early start of the century. In particular the magnetic field strength, electric field forces, and electromagnetic waves were investigated in more depth. In addition, the force of gravity or the gravitational forces of the impact of small and large particles were determined theoretically. Maxwell's electromagnetic field equations and the forces of positive and negative can be determined and will not be unipolar. 18. Towards the end of the century was known as the finite speed of light. However, in relation to this issue no connection no mathematical equation. During this period, the particle model of light are discussed, debated are affected by gravity. Bending of light in relation to the Cavendish in 1794 did serious work on the subject and the detailed work of Soldner 1804 published with the speed of light. In this work the influence of gravity in Newton's theories merged and a pulse rate and bending of light was calculated. Any bending of light to the sun may be a hyperbolic theories developed in proportion to the distance. Other alternative view of the Newtonian mechanics, the mass of potential energy per unit based on the exchange of opinions has emerged [1].

Bending of light is related to the work of Einstein in 1911, reviewing the "On the Influence of Gravitation on 
the Propagation of Light", published the article. This is the work of errors in 1915 and became a theory of general relativity finished or defined as the theory of relativity. Later the study of light passing through the holes in the back scattering of land as a result of the light $3 \mathrm{~m}$ diameter spiral-shaped bent theory has emerged. That is a very large gravity rays passing close to a certain extent bending emerged. In subsequent years, the photon model of light on the theories related to the bending of light produced by studies. The theory of relativity theory in 2004 and 1919 studies VBLI interferometer experimentally verified with very little error [1].

Schwarzschild coordinate system works by establishing their own land known as the de-adhered with the expansion of the white holes worked. The white holes in the universe may be a result of these studies, the idea emerged that the anti-truck. Developed the theory that the holes in the light into bending spiral [1].

In recent years the theory of quantum classical light Feynman revealed variable and related theorems unexplained parts brought a new openness in theory is developed. These studies provided above, Kevin Brown, "Reflections on Relativity" published by MathPages2010 are different theories about the behavior of light in his work.

In the linear and homogeny environments, Snell equitation was provided, considering the geometric optic. The theorems of the linear and nonlinear light are provided in the references [1-6].

Guided bend the laser beam in the magnetic field in 1985, C. A. Frost, S. L. Shope, R. B. Miller, G. T. Leifeste and C. E. Crist. IEEE Transaction on Nuclear Science, Vol. NS-32, No. 5 October Article was published in 1985, was carried out.

Some of the existing theories of physics can be counted as; quantum mechanics, quantum electrodynamics, chroma-quantum electrodynamics, $\mathrm{K}$ and $\mathrm{M}$ models, supersymmetry, superstring, anti-symmetry, Higgs model, symmetry breaking, the weak and strong nuclear field models [7-20].

\section{THE DIVISION AND BENDING OF GREEN AND RED SEMICONDUCTOR LASER}

In this study, the following pictures were taken using a transparent glass lens in the atmospheric pressure and room conditions. The special environment (laboratory) was not established when these pictures were taken. Bending was carried out at the experiment owing to the lenses used in laser light. We have named the "BENDING" as this behavior of Laser light. This term is not used in a very specific purpose. This is only a name that given to the event.
The experiment has been tested at the room temperature and normal atmospheric pressure. Laser source is used as the source of the two species. They are green and red laser with output power of $1 \ldots 5 \mathrm{~mW}$, with two standard AAA 1.5 V alkaline dry battery-operated hood "CLASS III" semiconductor laser diode sources. These laser sources which are not commercially traded electronic components. For this reason, laser diode sources are not produced by a very specific purpose.

Also a method has been developed to be seen the bending. This method is named as a method of briefly "RY". In this method, the laser light source, the lens is turned 90 degrees to the direction of the light reflected to the flat surface after the bending seen in the shape of the light is provided. The images in the article have been taken with a digital camera by RY method. These pictures were obtained by using different lenses and lens arrays. The distance between the laser source and the optical lens is a range between $4-400 \mathrm{~mm}$ and the distance between optical lens and the plane of rotation of the reflector is $4500 \mathrm{~mm}$.

The following Figures $\mathbf{1}$ and $\mathbf{2}$ were obtained using a red laser source. Using a single laser source and a single lens in the form of bending the bow bent to the left in Figure 1. Also using the same single source to be larger than the right and left small two different opposite process of bending and laser light was split into two in Figure 2. These two-piece lenses were used for the bending process. The different sizes request is possible to perform bending.

In Figure 3, the green semiconductor laser diode using the process of bending and laser light was split into two. One one-piece lens to divide the laser source was used. Laser light is divided in two opposite directions, and bending was the only lens.

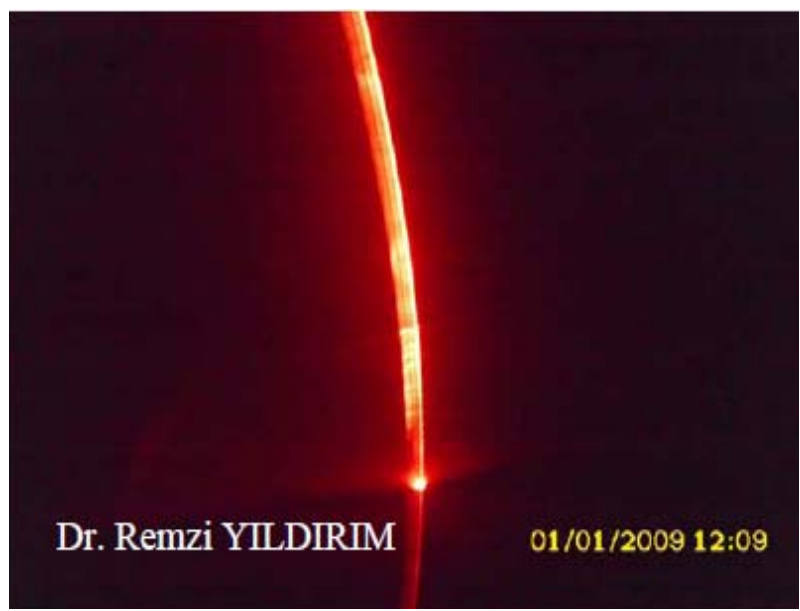

Figure 1. The red laser light bending to the left with one-piece lens. 


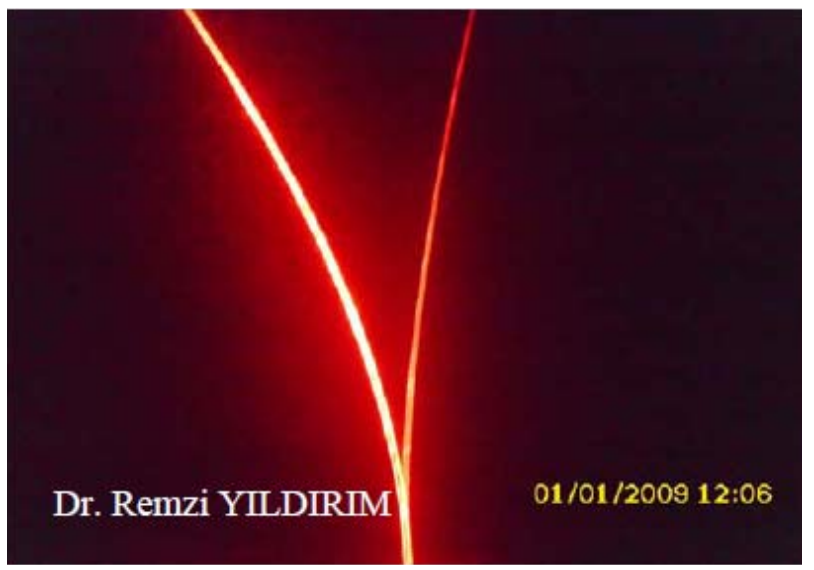

Figure 2. The red laser light deflected to the left to right with two lens.

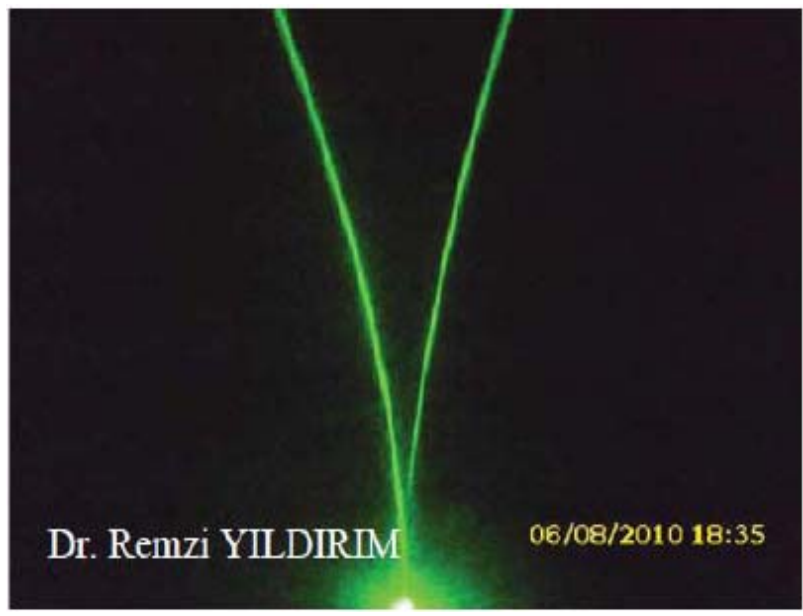

Figure 3. The green laser light using a single lens and the rightleft split into two opposite bending.

Figures 4 and $\mathbf{5}$ were obtained by using the same laser source. The number used is the one source. However, the number of lenses used in Figures 4 and 5 for 3 to 5 pieces of the lens used. Thus, the laser light from a single source is divided into three and five. At the same time, divided-light flat (center) and right-left bending process performed the same lenses. Laser light is only possible to bend to the right or left. Theoretically, the number of the laser light splitting can be increased until the distance between the adjacent the laser beams become zero. Splitting and bending should be-used in the lenses of the same features.

Figure 6 is also different from the red laser diodes using a lens from a single source laser beam bent in the form of parabola. Bending process used for the single lens. Figure 7 also is obtained by using the same lens from the green laser source; laser light was bent in the form of corner roundness.

Figure 8 is also used in the $6-7$ one-piece lens in the picture by using the green laser source-adjusted, were obtained from the bending of the laser beam. Laser light was bending as part of the oval shape.

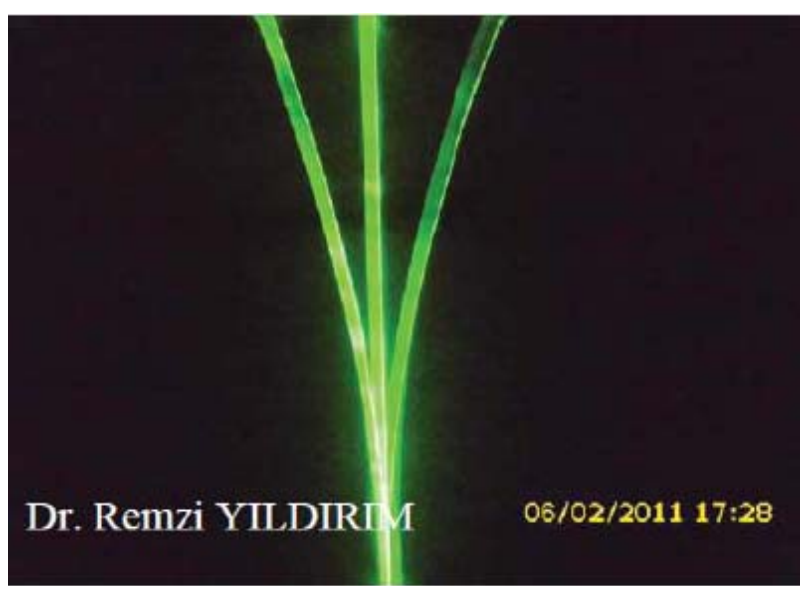

Figure 4. The green laser light is divided into three three-piece lens with a flat and right-left bending of light.

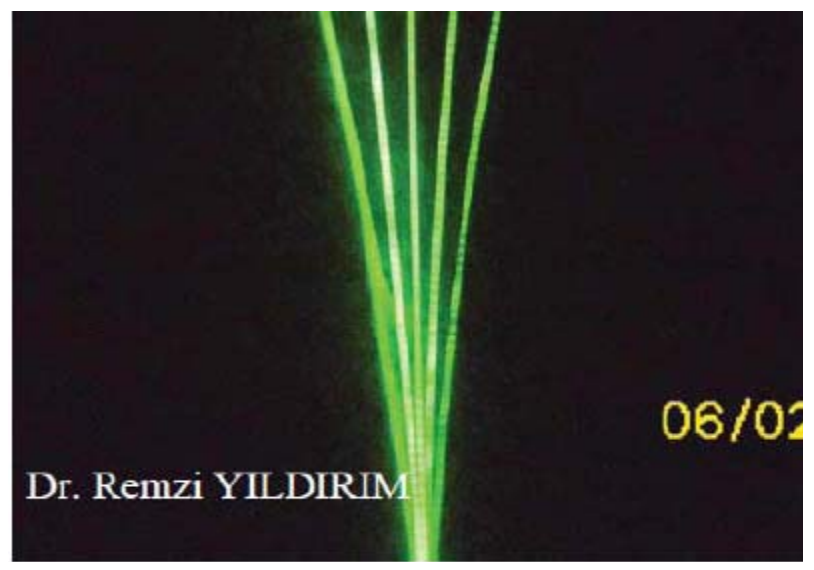

Figure 5. The green laser light lens, five, five, by dividing the straight and right-left bending.

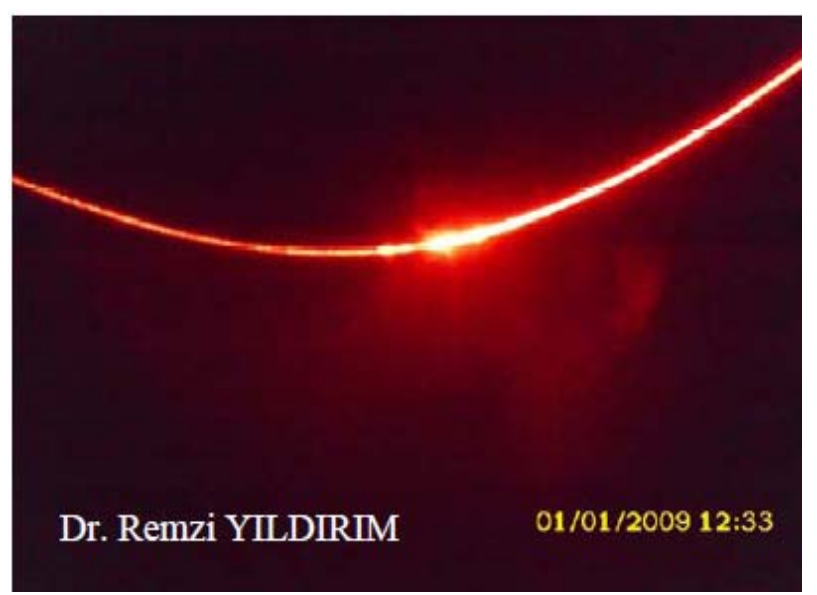

Figure 6. One-piece lens with the red laser light bending in a parabola. 


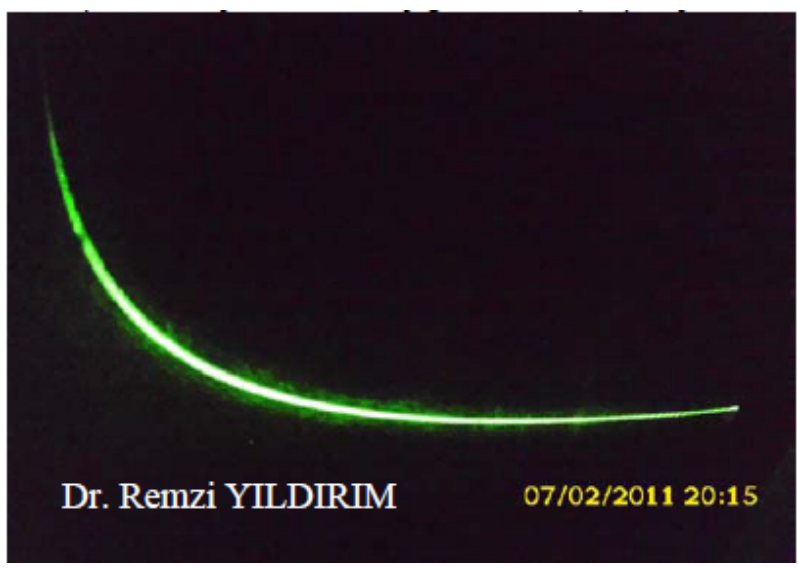

Figure 7. The green laser light deflected corner with one-piece lens.

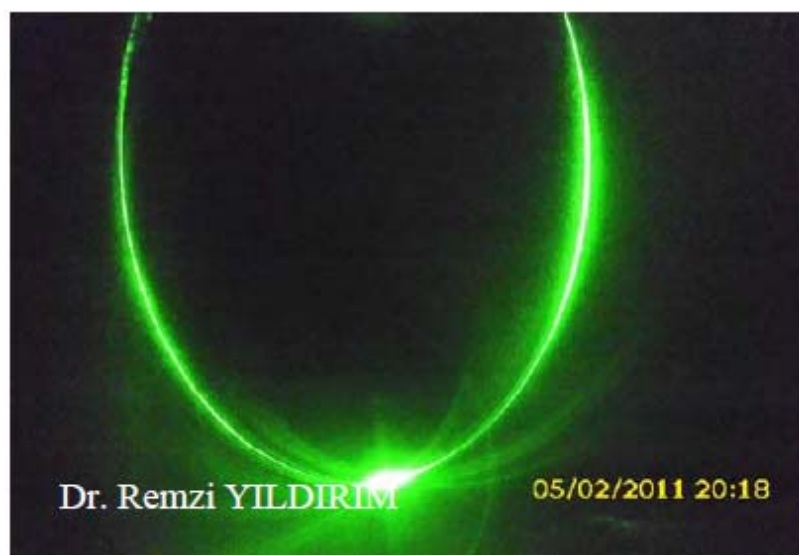

Figure 8. Obtained from a single laser source with an oval piece of green laser light and a single-piece lens.

Figures 9 - 10 are also one of the green laser light source as a bent part of the circle. Figure 10 also using the same lens has been bent in the form of various parts of the circle. Single lens is used for bending operations.

Figure 11 is also obtained from a single laser light source, green laser, were obtained using two different lenses. The laser light is divided into 4 parts, where the two laser light bending in the opposite direction, one right and the right part are obtained. It was carried out as part of the spread of laser light. Thus, preventing the spread of laser light at random and control the spread of the desired extent is provided. As a result, the single laser light source is divided into four parts and bent.

According to the particle theory of light, it is accepted that light consists of tiny particles called photons. In addition to that laser light propagates vectorally. The bending of laser light can not be explained with the available theories. It may be due to the new structure of light or a new undefined subatomic particle or particles that is not mentioned before. In our opinion, the light has a flexible symmetrical structure and this structure is

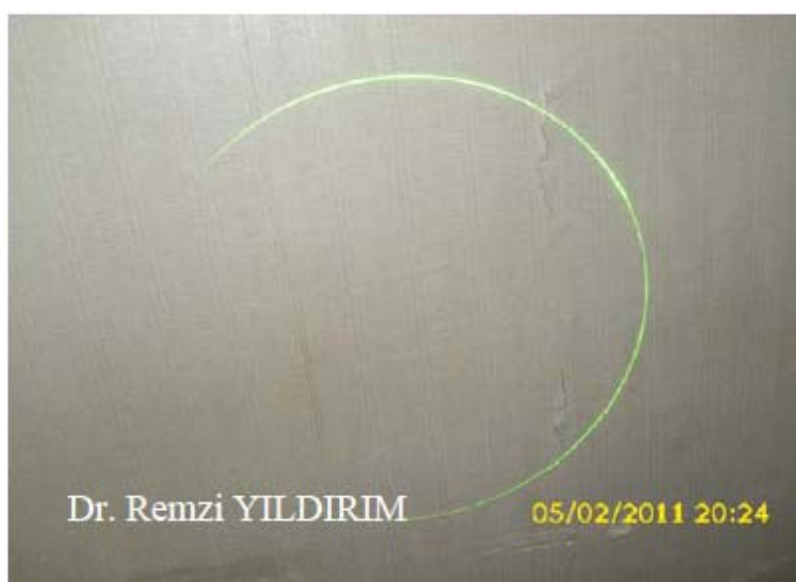

Figure 9. The green laser light bending as a part of a circle with one-piece lens.

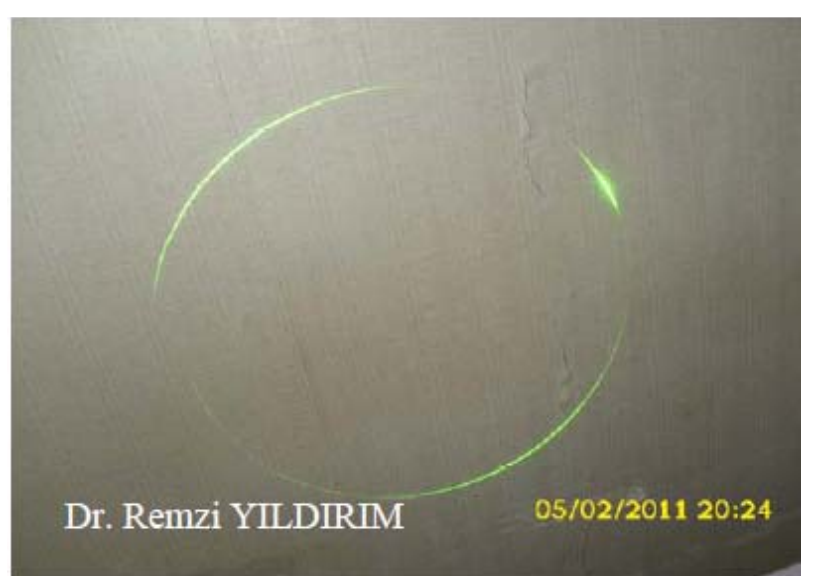

Figure 10. The green laser light bending as parts of a circle with one-piece lens.

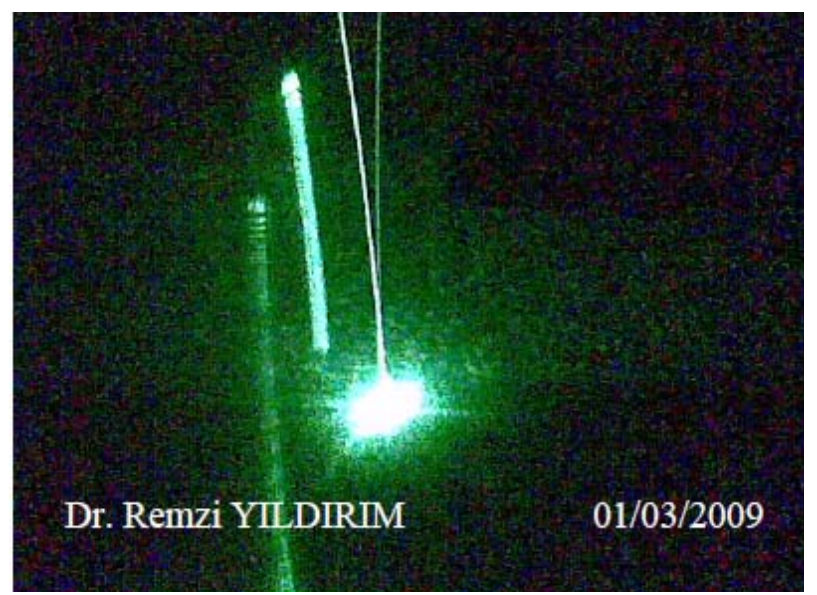

Figure 11. The single green laser light that is obtained from the laser source separation and different deflected in four parts.

destroyed with the lenses used in the experiment. For this bending to be permanent, permanent deterioration in the structure of the light should be achieved. This 
deterioration is a physical one instead of a chemical without changing the wavelength. At this stage, the mathematical and physical model of this situation is trying to be constructed.

\section{CONCLUSIONS}

This experimental study has been performed at atmospheric pressure, room temperature, and no electric field, magnetic field and polarization under laboratory conditions. The semiconductor laser as a source of green and red laser diode is used. The reason for the use of different laser sources was to see the effect of different wavelength lasers. Made of transparent glass lenses used in the experiment. There are no special-purpose optical filtering properties. During the trial, a different laser sources, for example, gas, liquid, free-electron, semiconductor, solid, paint or any laser light from the laser source is available.

The laser beam is chosen to be the better time for compliance is preferred, according to other natural lights in the experiment. Ideally, as the source of the use of single-mode laser may be better.

The lights that are obtained from the natural sources can bend. However, natural or derived from sources other than laser-guided-wave format, such as the introduction of laser light and the beam diameter must be adjusted. These conditions are provided; the normal bending of light can also be performed.

The properties of the lens that is used in experimental study as follows:

Transparency should be theoretically $100 \%$. Color as a transparent glass or transparent features of the refractive index close to the glass may be selected as other materials. In the case of color, used to absorb the energy of the laser beam wavelength and optical power, weakening the color should not be selected. If the optical filter is required it should be done before bending. In addition, the strong laser beam and temperature resistant to withstand the feature not to be. Not change the lens gets hot property. The quality of the lens surface roughness of the laser light is at least $\lambda / 4$, or optics to be used or is higher than $\lambda / 20$ should be preferred. Other measures should be commensurate with the wavelength of the laser used in the lens.

The lens must be on the body or bodies' carrier that are made by transparent glass. This body can also be used as part of the lens stalk as a stabilizer during assembly. Fit for purpose as part of the lens body in different ways. Stem and part of the quality of the lens body, the lens in any way affect the work function. However, during installation will cause excessive stretching and squeezing the lens part of the homogeneous structure of the lens is broken. Therefore, this situation should be considered during installation. The quality or accuracy of the surface is sufficient for the purpose of this section.

Intensity distribution of laser light is bent due to some technical reasons related to our work continues to change.

Fields of the applications are optical systems, optoelectronics, wireless optical communications, laser systems, aerospace, medical, industrial, aerospace, military areas, and optical measuring instruments.

In this study, the division of laser light and to make further comments in relation to bending and is thought to be too early to state your opinions. However, subject to the physical description and mathematical model to facilitate analysis of many experiments and observations suggest that there is need. We think this behavior is not linear laser. In this study the characteristic behavior of laser light known also think that's added a new feature. In this experiment, only the results obtained from the scientific community have made the determination we have opened the discussion with the share issue. Scientists that are interested in the issue of all the necessary support, suggestions and criticisms are waiting.

\section{REFERENCES}

[1] Brown, K. (2009) Relativity on reflectivity. Cambridge, London.

[2] Goca, N. (1996) Optics. Culture Press, Erzurum.

[3] Ghatak, A. (1986) Optics. Tata McGraw Hill, New Delhi.

[4] Hecth, E. (2002) Optics. 4th Edition, Addison Wesley, Boston.

[5] Yariv, A. (1989) Quantum electronics. 3th Edition, John Wiley and Sons, New York.

[6] Verdeyen, J.T. (2000) Laser electronics. Prentice Hall Inc., Upper Saddle River.

[7] Weinberg, S. (1983) The discovery of subatomic particle. Luis Weinberg and Weinabet Weinberg Trust, Cambridge.

[8] Greene, B.R. (1999) Superstrings hidden dimensions, and the quest for the ultimate theory. Norton, New York.

[9] Weinberg, S. (1992) Dreams of a final theory. Pantheon, New York.

[10] Kane, G. (2000) Supersymmetry/squarks, photinos, and the unveiling of the ultimate laws of nature. Parseus Books Group, New York.

[11] Greene, B. (2009) Fabric of cosmos. Penguin Press Science, New York.

[12] Feynman, R. (1988) QED: The strange theory of light and matter. Princeton University Press, Princeton.

[13] Hoddeson, L., Brown, L., Riondan, M. and Dressen, M. (1997) The rise of standart model. Cambridge University Press, Cambridge. doi:10.1017/CBO9780511471094

[14] Witten, E. (2001) Is supersymmetry really broken? hep-th/9409111, IASSNS-HEP-94-72.

[15] Di S.R., Notes on the conceptual of supersymmetry. Stony Brook University, New York. 
[16] Feynman, R. (1988) Superstrings: A theory of everything? Cambridge University Press, Cambridge.

[17] Nambu, Y. (1985) Quarks frontiers in elementary particle physics. World Scientific Pub. Co. Pte. Ltd, Singapore City.

[18] Raman, V.C. (1999) Nobel lectures. Physics 1922-1941, Elsevier Publishing Company, New York.
[19] Higgs, P.W. (1964) Broken symmetries and the masses of gauge bosons. Physical Review Letters, 13, 508-509. doi:10.1103/PhysRevLett.13.508

[20] Higgs, P.W. (1964) Broken symmetries massless particles and gauge fields. Physical Letters, 12, 132-133. doi:10.1016/0031-9163(64)91136-9 